\title{
The conserved AAUAAA hexamer of the poly(A) signal can act alone to trigger a stable decrease in RNA polymerase II transcription velocity
}

\author{
ANITA NAG, KAZIM NARSINH, AMIR KAZEROUNINIA, and HAROLD G. MARTINSON \\ Department of Chemistry and Biochemistry and the Molecular Biology Institute, University of California at Los Angeles, Los Angeles, California \\ 90095-1569, USA
}

\begin{abstract}
In vivo the poly(A) signal not only directs $3^{\prime}$-end processing but also controls the rate and extent of transcription. Thus, upon crossing the poly(A) signal RNA polymerase II first pauses and then terminates. We show that the G/U-rich region of the poly(A) signal, although required for termination in vivo, is not required for poly(A)-dependent pausing either in vivo or in vitro. Consistent with this, neither CstF, which recognizes the G/U-rich element, nor the polymerase CTD, which binds CstF, is required for pausing. The only part of the poly(A) signal required to direct the polymerase to pause is the AAUAAA hexamer. The effect of the hexamer on the polymerase is long lasting-in many situations polymerases over $1 \mathrm{~kb}$ downstream of the hexamer continue to exhibit delayed progress down the template in vivo. The hexamer is the first part of the poly(A) signal to emerge from the polymerase and may play a role independent of the rest of the poly $(A)$ signal in paving the way for subsequent events such as $3^{\prime}$-end processing and termination of transcription.
\end{abstract}

Keywords: transcriptional pausing; cleavage and polyadenylation; poly(A)-dependent termination; poly(A) signal; RNA polymerase; CstF

\section{INTRODUCTION}

Most eukaryotic mRNAs are cleaved and polyadenylated in the nucleus prior to export into the cytoplasm. Although the poly(A) tail performs critical regulatory functions in the cytoplasm, it is increasingly clear that the cleavage and polyadenylation process itself is equal in importance to the function of the tail per se. For example, although cleavage at the poly(A) site could, in principle, be carried out by a single protein, a complex array of 20 or more polypeptides, including even the polymerase, collaborate to carry out this function (Gavin et al. 2002; Calvo and Manley 2003; Proudfoot 2004). Such a high degree of complexity for such a simple task reflects the fact that the cleavage and polyadenylation apparatus makes intimate connections to all major aspects of mRNA and its production from the $5^{\prime}$ cap to transport-including splicing, transcription termi-

Reprint requests to: Harold G. Martinson, Department of Chemistry and Biochemistry and the Molecular Biology Institute, University of California at Los Angeles, Los Angeles, CA 90095-1569, USA; email: hgm@chem.ucla.edu; fax: (310) 206-4038.

Article published online ahead of print. Article and publication date are at http://www.rnajournal.org/cgi/doi/10.1261/rna.103206. nation, cleavage and polyadenylation (of course), and possibly even transcription initiation and/or reinitiation (Dantonel et al. 1997; Flaherty et al. 1997; Hammell et al. 2002; Maniatis and Reed 2002; Proudfoot et al. 2002; Calvo and Manley 2003; O'Sullivan et al. 2004; Ansari and Hampsey 2005). It is likely that poly(A) signal activity has been designed by the cell to serve as a major checkpoint that integrates the various aspects of pre-mRNA processing with both termination of transcription and preparations for transport (Orozco et al. 2002; Bird et al. 2005).

In this article we focus on the manner in which the poly(A) signal affects transcription. The poly(A) signal dictates changes in both the rate (pausing) and the processivity (termination) of transcription by RNA polymerase II. However, the mechanism by which the poly(A) signal contributes to these processes is not known. For example, the $5^{\prime} \rightarrow 3^{\prime}$ exonuclease Rat1 contributes to poly(A)-dependent termination in yeast, consistent with a model in which the role of the poly(A) signal is to cleave at the $\operatorname{poly}(\mathrm{A})$ site so as to provide the entry point for this termination factor (Kim et al. 2004b). At the same time, certain mutants in yeast that cannot cleave at the poly(A) 
site nevertheless undergo poly(A)-dependent termination (Sadowski et al. 2003; Zhelkovsky et al. 2006), suggesting that poly (A) site cleavage may not be a fundamental part of the poly(A)-dependent termination mechanism. In another example, the poly(A) signal in mammals can alone drive termination in a heterologous sequence background (Orozco et al. 2002), yet in their native context, some poly (A) signals require auxiliary elements to effect termination (Connelly and Manley 1989; Dye and Proudfoot 2001; West et al. 2006).

Recently, the core cleavage and polyadenylation factor Pcf11 has been found to exhibit constitutive RNA polymerase II release activity in minimal reconstituted systems from both yeast and flies (Zhang et al. 2005; Zhang and Gilmour 2006). If Pcf11 is involved in poly(A)-dependent termination, then the role of the poly(A) signal clearly includes the recruitment of this factor, but it remains unknown whether the first-order kinetics of basal poly(A)dependent termination (Orozco et al. 2002) reflects the rate of recruitment or the rate of activation of Pcf11. Interestingly, Pcf11 transcript release activity requires a paused polymerase (Zhang et al. 2005; Zhang and Gilmour 2006). Therefore, it is possible that the intrinsic ability of the poly(A) signal to pause the polymerase (Orozco et al. 2002; Park et al. 2004) is related to activation of the Pcfl1 transcript release activity.

We are interested in the basal mechanism by which the poly(A) signal drives both pausing and termination, with the focus in this article on pausing. Figure $1 \mathrm{~A}$ is a cartoon of the core interactions in the mammalian cleavage and polyadenylation apparatus. The two core elements of the poly(A) signal, the AAUAAA hexamer and the G/U-rich region, are recognized by CPSF and CstF, respectively (Zhao et al. 1999). CPSF and CstF bind to each other specifically and to the poly(A) signal cooperatively (Gilmartin and Nevins 1989; Murthy and Manley 1995; Takagaki and Manley 2000). CstF also binds the carboxyl-terminal repeat domain (CTD) of the RNA polymerase II large subunit (Fong and Bentley 2001). The CTD is required for both poly(A) site cleavage (Ryan et al. 2002; Bird et al. 2004) and for poly(A)-dependent termination (Park et al. 2004; Zhang et al. 2005), but it is not required for pausing (Park et al. 2004). It is known that the poly(A) signal hexamer is required for poly(A)-dependent pausing (Orozco et al. 2002; Park et al. 2004), but the lack of a CTD requirement raises the question (see cartoon in Fig. 1A) of whether CstF or even the G/U-rich region of the poly(A) signal might likewise not be required. If so, this would suggest a stepwise assembly model for the cleavage and polyadenylation apparatus in which the first part of the poly(A) signal to emerge from the polymerase directs it to pause, whereupon the second part recruits additional proteins that combine with the first to drive termination.

To investigate the basal mechanism of poly(A)-dependent pausing and termination, we placed poly $(\mathrm{A})$ signals

A

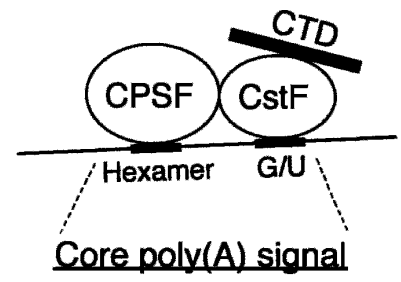

B

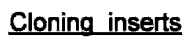

(i) AACAMATAMAGCATTTTTTTCACTGCATTCTAGTTGTGONTERG (ii) AACARgTACEGCATTTTTTCACTGCATTCTAGTTETGOTITGRG

(iii) AACMMTMMaGCTTAGATCTCGAGACACAACCAAGCATTMATMMG (iv) AACAMrattGCTTAGATCTCGAGACACAACCAAGCATTaMrana

(v) AACAATattGCTTAGATCTCGAGACACAACCAAGCATTatcgatG

Polv(A) with G/U core poly(A) signal core with mutant hexamer GN-less polv(A) two hexamers one hexamer no hexamer

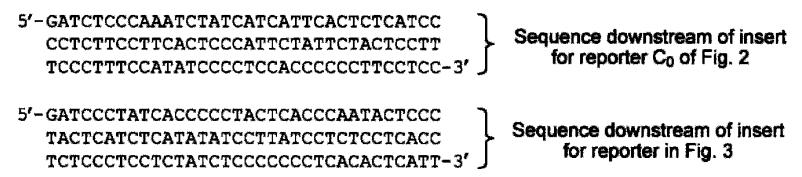

FIGURE 1. The $\operatorname{poly}(A)$ signal core and G/U-less variants thereof. (A) The core of the polyadenylation apparatus (Zhao et al. 1999; Proudfoot 2004). (B) Sequence elements used as inserts in the reporter constructs of this work. There is no G/U-rich region downstream of the insert.

into foreign sequence backgrounds, taking particular care to ensure that DNA downstream of the poly(A) signal was devoid of any natural elements that might modify poly(A) signal activity (Tran et al. 2001; Orozco et al. 2002). It was such experiments that previously showed that polymerase density first rises and then falls after crossing the poly(A) signal, reflecting poly(A)-dependent pausing of the polymerases followed by release (Orozco et al. 2002). The fact that pausing and termination can be functionally uncoupled by removal of the polymerase CTD (Park et al. 2004) suggests that pausing may be more than merely a precursor to termination, and may instead be a functionally discrete activity. Here we report that whereas the entire poly(A) signal is required for poly(A)-dependent termination, only its initial portion (the hexamer) is required for pausing, the first detectable effect of the poly(A) signal on transcription.

\section{RESULTS}

\section{The G/U-rich region of the poly(A) signal is not required for poly(A)-dependent pausing by RNA polymerase II in vivo}

To assess the role of the G/U-rich region in poly(A)dependent pausing, we transfected plasmids containing intact or mutated poly (A) signals into COS cells and then measured polymerase occupancy on the template downstream of the poly(A) element by means of run-on 
transcription (Orozco et al. 2002; Park et al. 2004). Slowing or pausing of transcription results in an increase in polymerase occupancy at steady state (hereafter referred to as polymerase density) due to the longer dwell times of the polymerases at one or more positions on the template.

Polymerase density was measured by using G-less cassettes. The elements to be assayed were placed in reporter plasmids between two G-less cassettes and then transfected into cells. Nuclei were isolated, run-on transcription was carried out in the presence of $\left[\alpha-{ }^{32} \mathrm{P}\right] \mathrm{CTP}$, the resulting RNA was digested with RNase T1, and the surviving G-less cassettes were displayed on a gel. The number of poly- merases in each cassette on the template is proportional to the intensity of each corresponding RNA band on the gel. Using the reporter series shown in Figure 2A, in which the downstream cassette was placed at increasingly greater distances from the poly(A) signal, Orozco et al. (2002) detected an increase in polymerase density in the downstream cassette (pausing) when it was close to the poly (A) signal, but exponentially decreasing polymerase densities (termination) as the cassette was placed increasingly farther away. Results obtained by using cassettes (Yeung et al. 1998; Steinmetz and Brow 2003; Zhelkovsky et al. 2006) are comparable to those obtained by slot blot hybridization

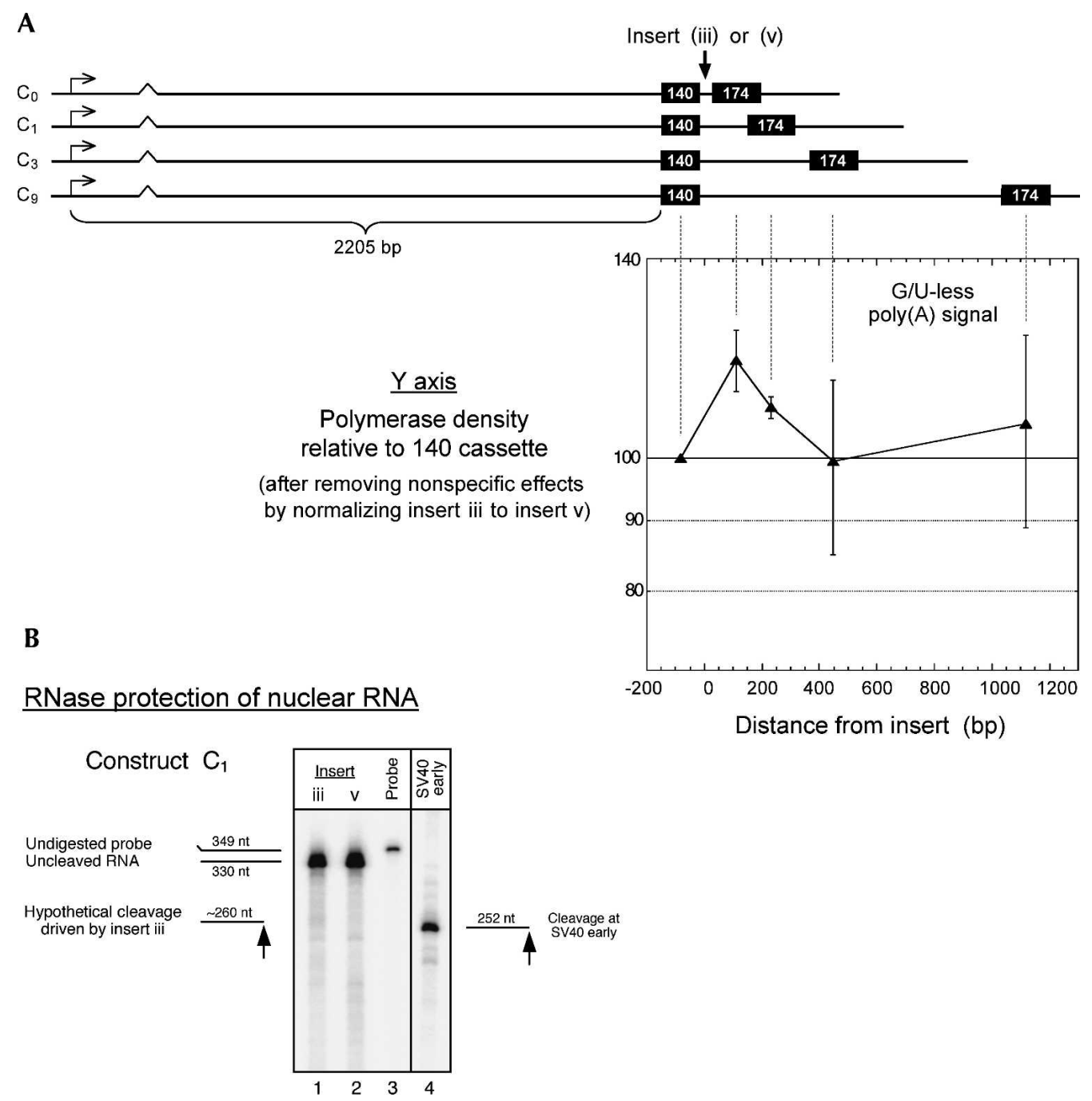

FIGURE 2. The G/U-rich region of the poly $(A)$ signal is not required for pausing. (A) Pausing occurs in the absence of a G/U-rich region. The reporters used are identical to the corresponding $\mathrm{A}_{3}\left\langle\mathrm{EC}_{\mathrm{n}}\right\rangle$ constructs of Orozco et al. (2002) except that they contained inserts iii and $v$ of Figure $1 \mathrm{~B}$ instead of a poly $(A)$ signal (also an AATAAA hexamer centered 65 bp upstream of the 140-bp cassette was removed by converting it to CCCGGG by site-directed mutagenesis). The region upstream of the 140-bp cassette contains the SV40 small intron and is mostly identical to the first two-thirds of the $A_{3}$ region described in Orozco et al. (2002). The upstream cassette here is 140 bp rather than the 136 bp of Orozco et al. (2002) because of the different cloning junctions. The results plotted are the average and range from two different experiments. (B) Processing does not occur in the absence of a G/U-rich region. RNase protection was carried out on nuclear RNA. The probe (lane 3) for the G/U-less constructs in lanes 1 and 2 was generated by RNA polymerase T3 transcription of a PCR fragment generated from construct $\mathrm{C}_{1}(A$, top $)$ containing insert iii (Fig. 1B). The RNase protection in lane 4 for a poly $(A)$ signal was as in Park et al. (2004). In both cases only T1 RNase was used. 
(Yeung et al. 1998; Dichtl et al. 2004), but cassettes offer various theoretical and practical advantages, as discussed previously (Yeung et al. 1998; Orozco et al. 2002).

Figure $1 \mathrm{~B}$ shows the cloning inserts that we have used to evaluate the role of the G/U-rich region. Insert $i$ is a core wild-type poly(A) signal, and insert ii is its hexamer mutant. Inserts iii and iv are G/U-less poly(A) signals. Insert iii has two hexamers and was included because some poly(A) signals contain two closely spaced hexamers (Connelly and Manley 1988; Natalizio et al. 2002). Insert iv contains a single hexamer and is identical to insert iii except that the upstream hexamer in insert iii was mutated. Elements with one and two hexamers, we have found, behave similarly (e.g., Figs. 3B, 4B), so we have used them interchangeably. Insert $\mathrm{v}$ is a control for hexamers iii and iv in which both hexamers have been mutated.

Figure $2 \mathrm{~A}$ shows that poly(A)-dependent pausing occurs for a $\operatorname{poly}(A)$ signal that is completely devoid of a $G / U$-rich region. For the experiment of Figure 2A, the same reporters as for Figure 5C of Orozco et al. (2002) were used except that a G/U-less, rather than a complete, poly(A) signal was assayed. Following the approach of Orozco et al. (2002) the G/U-less poly (A) signal to be assayed (Fig. 1B, insert iii) was run in parallel with an identical G/U-less poly $(A)$ signal whose hexamers had been inactivated by mutation (Figure $1 \mathrm{~B}$, insert $\mathrm{v})$. The results show that the polymerase density increases downstream of the G/U-less poly(A) signal despite the fact that its only noticeable resemblance to a normal poly(A) signal is the presence of two AAUAAA hexamers. Therefore, the G/U-rich region of the poly(A) signal is not required to trigger pausing by the polymerase over downstream DNA. Although there is substantial scatter over the far downstream locations of the template in these experiments, it is clear that the G/U-less poly(A) signal, as expected, elicits little if any transcription termination (reflecting the requirement of the G/U-rich region for transcription termination) (see Connelly and Manley 1988; cf. to Fig. 5C of Orozco et al. 2002 or to Figs. 2, 4A of Park et al. 2004).

It is unlikely that a cryptic $\mathrm{G} / \mathrm{U}$-rich region in the $\mathrm{C}_{0}$ and $\mathrm{C}_{1}$ reporter constructs accounts for the pausing downstream of the inserted elements in Figure $2 \mathrm{~A}$. Reporter $\mathrm{C}_{0}$, which gives the peak of hexamer-dependent pausing, has no discernible GT-rich region or any T patches (four out of five T's) (Chen et al. 1995) for $>100$ bp downstream of the insertion site (see Fig. 1B). However, construct $\mathrm{C}_{1}$ does harbor a patch of nucleotides $\sim 40$ bp downstream that bears a mild resemblance to a G/T-rich region. To assess the likelihood of cryptic G/U-rich region activity in construct $\mathrm{C}_{1}$, we used RNase protection to check for $3^{\prime}$ end cleavage in RNA from cells transfected with the $\mathrm{C}_{1}$ / insert iii construct. Lane 1 in Figure $2 \mathrm{~B}$ reveals little or no 3 '-end cleavage for the $\mathrm{C}_{1} /$ insert iii construct. However, when construct $C_{1}$ contains a poly $(A)$ signal (lane 4), efficient $3^{\prime}$-end cleavage occurs. Therefore, we conclude that the G/U-rich region is indeed functionally absent in our G/U-less constructs, and poly(A)-dependent pausing depends on the insert iii hexamers alone.

We decided to repeat the experiment of Figure 2A using a new reporter construct (Fig. 3A). In this reporter different sequences flank the test element insertion site, different sequences separate the G-less cassettes, and a single reporter contains several cassettes so that a single transfection replaces the multiple transfections previously required for a reporter series (Fig. 2A; Orozco et al. 2002).

First, we tested this reporter using an intact poly(A) signal. A typical result comparing an intact core poly(A) signal (insert $\mathrm{i}$ ) with its hexamer mutant (insert ii) is shown in the two gel lanes of Figure 3A. It can be seen that the polymerase density, as reflected in the cassette signals from the run-on transcription, is similar for both wild-type and mutant templates in the vicinity of the poly(A) signal. However, within a few hundred nucleotides of the poly (A) site, the cassette signal for the wild type has clearly grown greater than that for the mutant (cf. signals for the 174nucleotide [nt] cassettes), suggesting that the polymerases have slowed down in this region, thereby increasing their linear occupancy along the template. Still further down the template, in the vicinity of the 261-bp cassette, the cassette signal for the wild type is clearly much less than that for the mutant, reflecting the onset of poly(A)-dependent termination. The results from three such experiments are summarized in the graph (Fig. 3A).

The line in the graph of Figure $3 \mathrm{~A}$ represents the signal intensity for each cassette in gel lane $\mathrm{i}$ [for the wild type poly(A) signal], expressed as a percentage of the equivalent cassette in gel lane ii [for the mutant poly(A) signal]. Each value is also normalized to that for the 104-nt cassette so that the polymerase density immediately preceding the poly(A) site is defined as $100 \%$. The results confirm for this new reporter the transcriptional effect typically projected downstream by a poly (A) signal-polymerase density on the wild-type template first rises $>100 \%$, suggesting pausing or slowing down, and then it drops $<100 \%$ farther downstream, consistent with poly(A)-dependent release (Orozco et al. 2002).

The results for $\mathrm{G} / \mathrm{U}$-less poly $(\mathrm{A})$ signals (Fig. 1B, inserts iii and iv) are illustrated in Figure 3B. In this case the signal intensity for each cassette on the templates having one or two hexamers (inserts iv or iii) is expressed as a percentage of the equivalent cassette on the template in which both hexamers have been mutated (Fig. 1B, insert v). It can be seen that, as for Figure $2 \mathrm{~A}$, the $\mathrm{G} / \mathrm{U}$-less poly $(\mathrm{A})$ signals lead to an increase in polymerase density downstream, suggesting a slow-down or pausing of the polymerases. The results also show that one and two hexamers behave similarly for this aspect of poly(A) signal function.

Park et al. (2004) showed that the CTD is not required for poly(A)-dependent pausing. If hexamer-dependent pausing is equivalent to poly $(\mathrm{A})$-dependent pausing, then 

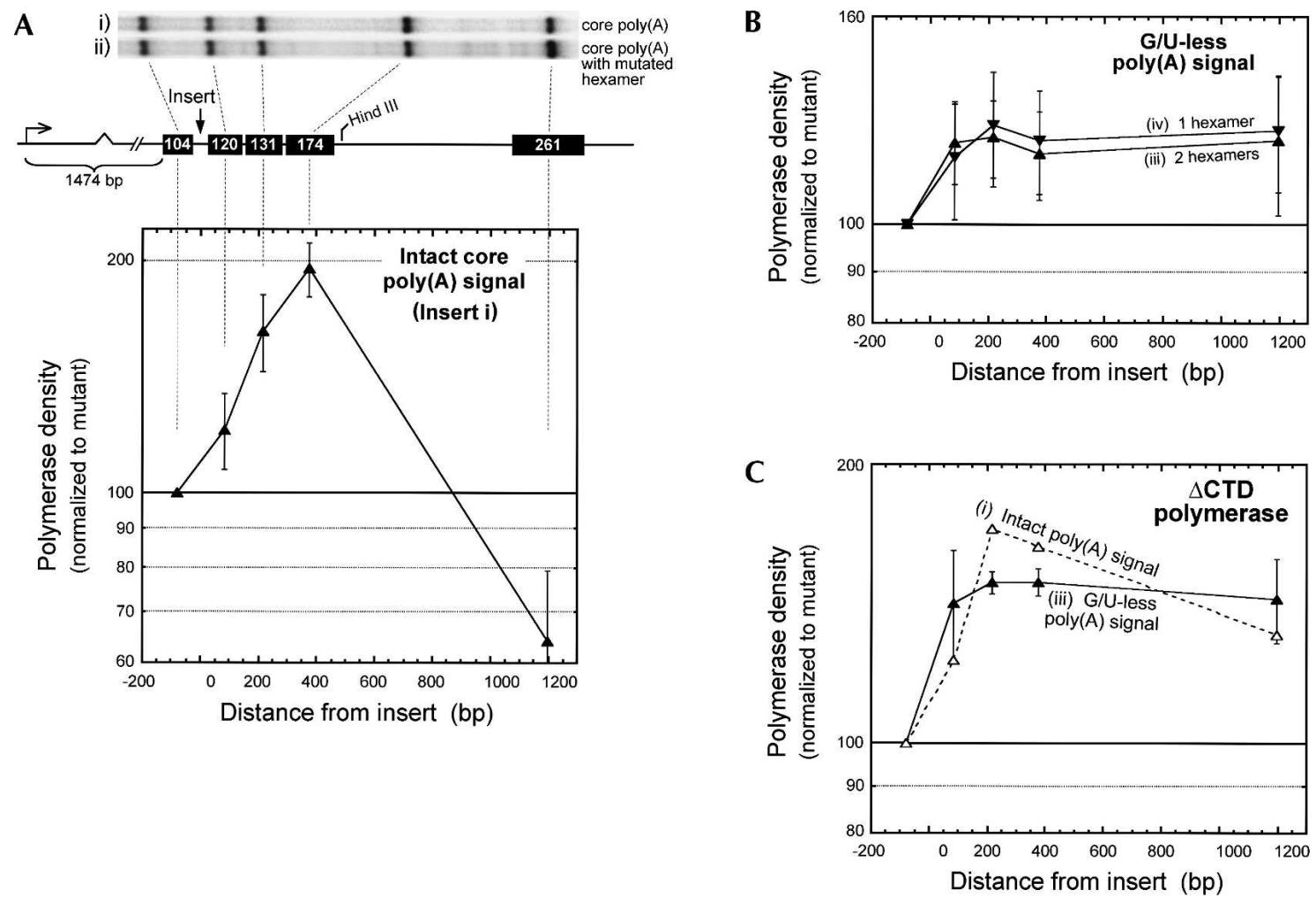

FIGURE 3. Pausing and termination with the penta-cassette reporter. (A) Intact poly $(A)$ signal. The gel lanes show results of representative G-less cassette run-on assays of nuclei isolated from COS cells transfected with reporter plasmids containing five G-less cassettes and either insert $i$ or insert $i i$ (centered at the position of the arrow). The reporter (roughly to scale) was derived from pAP (cat〉 of Figure 6A in Tran et al. (2001), to which it is identical downstream of the HindIII site. The region upstream of the 104-bp G-less cassette contains the SV40 small intron and is mostly identical to the first two-thirds of the $\mathrm{A}_{3}$ region described in Orozco et al. (2002). The graph summarizes the results of three experiments, showing the average and standard deviation. Because termination is exponential, we retain the convention of plotting the results semilogarithmically (Orozco et al. 2002). (B) G/U-less poly $(A)$ signals. The average and standard deviation of three (element iii) or the average and range of two (element $i v$ ) entirely separate experiments are shown. (C) $\Delta$ CTD polymerase. For methods, see text and Park et al. (2004). Closed triangles show the average and range for two different experiments with element iii (normalized to $v$ ).

it should similarly be CTD independent. To ask whether the CTD is required for hexamer-dependent pausing, we transfected COS cells with reporter plasmid together with an expression plasmid encoding a CTD-deleted Rpb1 (Park et al. 2004). This $\triangle$ CTD Rpbl also had an $\alpha$-amanitin resistance mutation, which allowed us to restrict transcription in the assay to the ectopically expressed Rpb1 by adding $\alpha$-amanitin to the run-on reaction (Fong et al. 2003; Park et al. 2004). Figure 3C shows the response of the $\triangle$ CTD polymerase to both an intact poly $(\mathrm{A})$ signal and to a $\mathrm{G} / \mathrm{U}$-less poly(A) signal. In agreement with previous results (Park et al. 2004), a full poly(A) signal drives robust and sustained pausing by the $\Delta$ CTD polymerase (Fig. $3 \mathrm{C}$, dashed line). The solid line in Figure $3 \mathrm{C}$ shows that the effect of a G/U-less poly(A) signal is equivalent. Therefore, we conclude, for a $\triangle$ CTD polymerase, that the poly(A) signal drives pausing via its hexamer.

Although the intact poly(A) signal and the G/U-less poly(A) signal affect the $\triangle$ CTD polymerase similarly (Fig. 3C), the magnitude of the pausing response differs between intact and G/U-less poly(A) signals when they operate on the endogenous polymerase having a full CTD (Fig. 3A,B). Perhaps the core mechanism of poly(A)-dependent pausing is based on a signal that is delivered from the hexamer to the body of the polymerase, but interactions involving the CTD can modify this response. Differences in the template may also modulate the response of the polymerase to the hexamer (cf. Figs. 2A and 3B). In summary, regardless of the details of the experiment, G/U-less poly(A) signals, having one or more hexamers, always elicit an increase in polymerase density for a significant distance downstream of their position on the template. We conclude that the AAUAAA hexamer, on its own, is able to direct a longlasting change in the polymerase that causes it to slow down or pause.

\section{The $G / U$-rich region is not required for poly(A)-dependent pausing in vitro}

We sought independent confirmation of the idea that only a portion of the poly(A) signal is sufficient to pause the polymerase. For this we turned to the in vitro transcription 
A

\section{$\underline{\text { In vitro transcription elongation assay }}$}

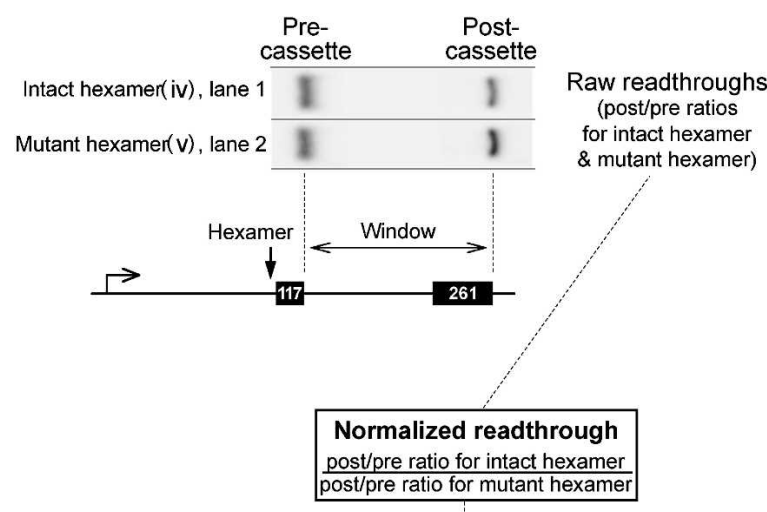

B

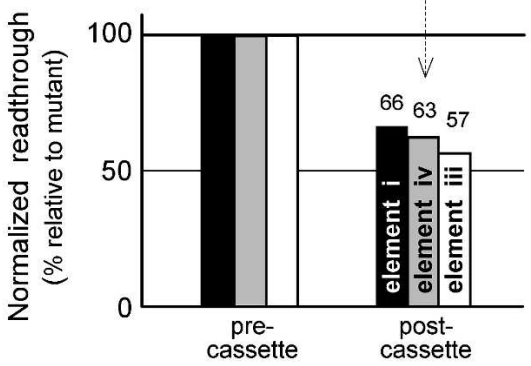

C

Size exclusion of ternary complexes

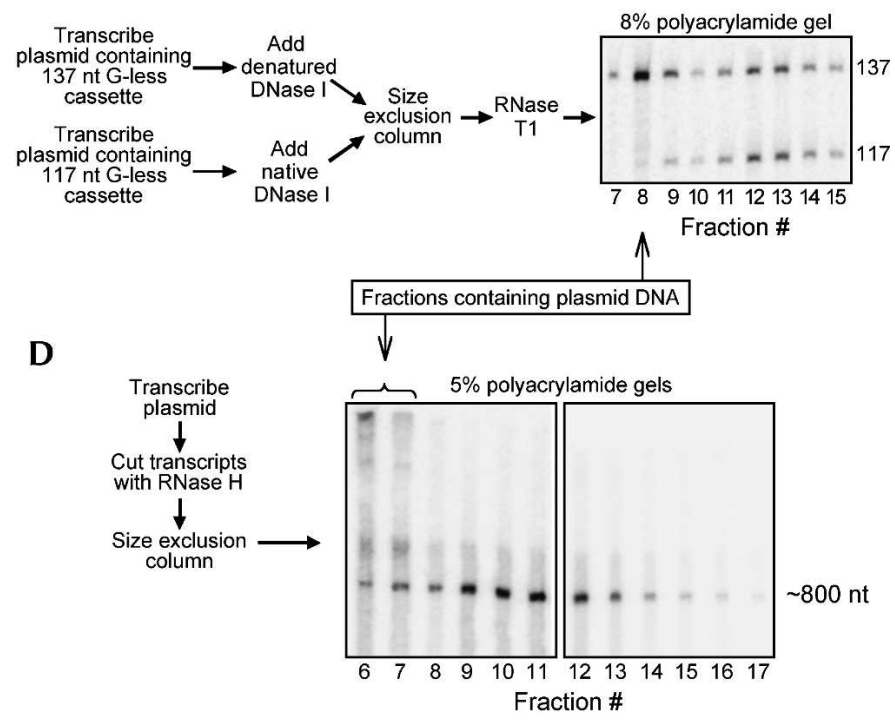

E

Pausing analysis of isolated ternary complexes

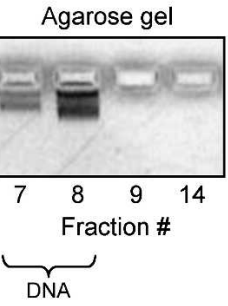

FIGURE 4. The G/U-rich region is not required for poly $(A)$-dependent pausing/slowing in vitro. $(A)$ The AAUAAA hexamer directs pausing in vitro. The gel lanes show representative results of an in vitro elongation assay with reporter plasmids containing either a single hexamer (insert $i v$ ) or its mutant (insert $v$ ) centered at the position of the arrow in the map beneath the gel lanes. The reporter map shown is the same as that for $\mathrm{pAP}\langle\mathrm{cat}\rangle$ in Figure 6A of Tran et al. (2001) except for the hexamer insert (there are additional irrelevant differences outside the region mapped). The percentage readthrough was calculated (see figure) by normalizing the post/pre ratio for element $i v$ to that for its mutant (i.e., element $v$ ). The result is shown in the middle bar of the Figure $4 \mathrm{~B}$ histogram. $(B)$ Hexamer-mediated pausing is comparable to pausing directed by a full poly $(A)$ signal. The percentage readthroughs are shown for a full $\operatorname{poly}(A)$ signal as well as for two different G/U-less constructs. The post/pre ratio for each element was normalized to that for its respective mutant ( $i$ normalized to $i i$; $i i$ and $i v$ normalized to $v$ ). ( $C$ ) Size exclusion of transcripts depends on the integrity of the DNA. Two plasmids, one expressing a 117-nt G-less cassette and the other a 137-nt cassette, were transcribed in parallel, and then either active (117 construct) or heat denatured (137 construct) DNase I was added to each mixture for 3 min. The reactions were stopped with salt, Sarkosyl, and EDTA; mixed together; and then loaded on a size-exclusion column. The RNA from each fraction was isolated, digested with RNase T1, and displayed on an $8 \%$ polyacrylamide gel. The experiment was repeated, but with the DNase I treatments reversed, with similar results. $(D)$ Size exclusion of transcripts depends on the tether to the polymerase. Transcription was carried out in the presence of a DNA oligonucleotide that directs RNase $\mathrm{H}$ to cut the transcript about $800 \mathrm{nt}$ downstream of the promoter (Rigo et al. 2005). Size-exclusion chromatography was then carried out to separate the ternary complexes from the released RNA. The column fractions were electrophoresed in two groups at different times, so the intensities of the two gels relative to each other may not exactly correspond. (E) Direct evidence that ternary complexes slow down after crossing an AAUAAA hexamer. Elongation assays were carried out on reporters containing either element iii or element $\mathrm{v}$ of Figure 1B, and the transcription mixtures were then fractionated by size-exclusion chromatography. An aliquot of each fraction was analyzed for plasmid DNA by agarose gel electrophoresis to identify the fractions containing ternary complexes. These fractions $(7$ and 8$)$ were then analyzed by using the methods of $A$ to determine what proportion of ternary complexes had reached the end of the post-cassette (and, conversely, what proportion still remained within the "window"). The error bars represent the range of values from two separate experiments. The reporter used is the same as in Figure 5, A and B, but with a G/U-less element in place of the poly $(A)$ signal.

elongation assay of Tran et al. (2001). This assay determines the efficiency with which polymerases can travel from one G-less cassette to another during a limited period of time after crossing a poly(A) signal. If the polymerases travel more slowly (or pause) between the cassettes, fewer of them will reach the downstream cassette during the time 
A Western Blot

Cleavage of pre-made RNA
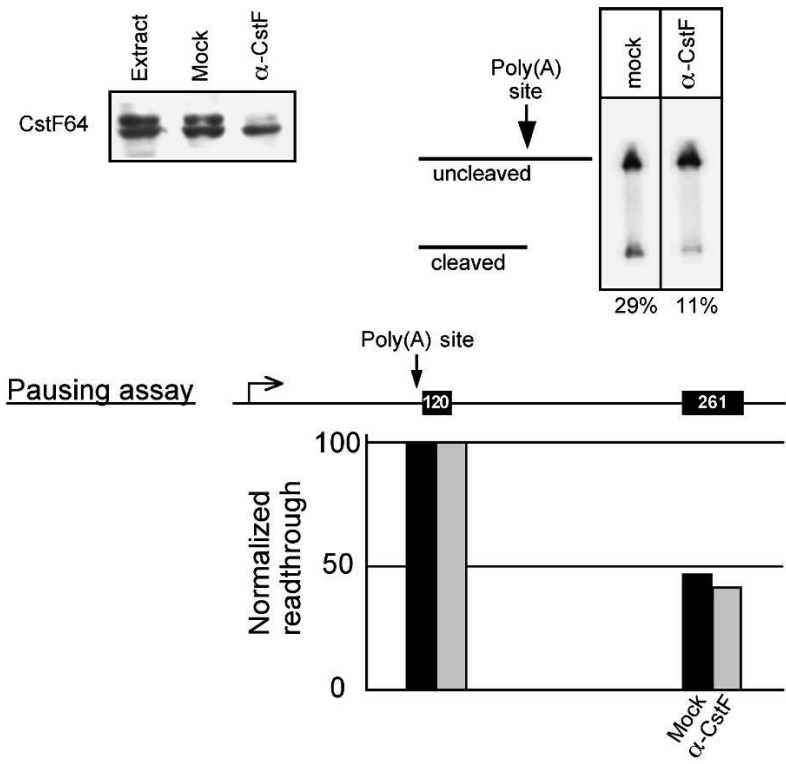

B

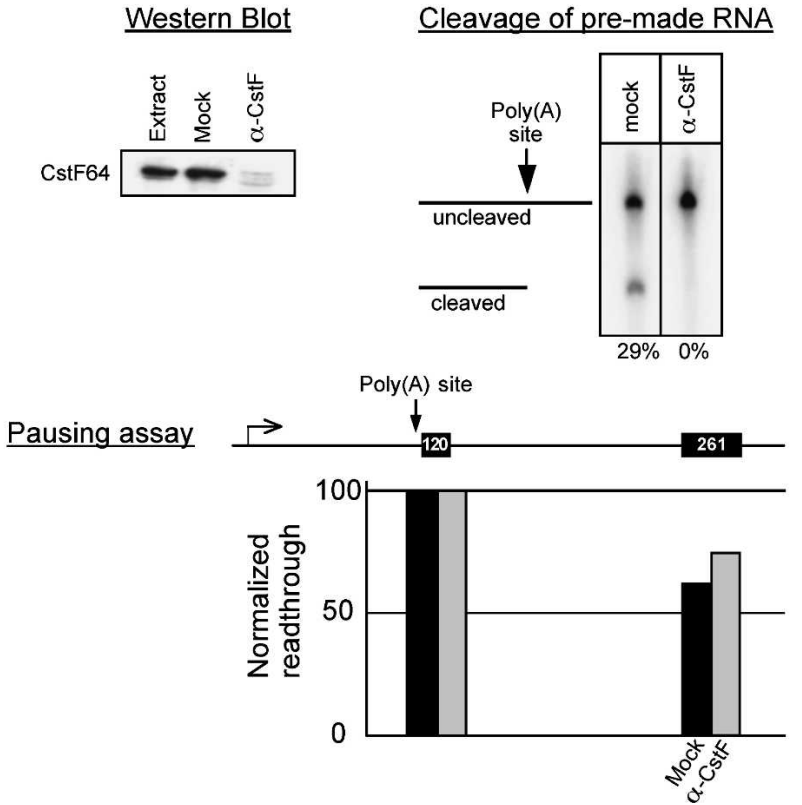

C

Western Blot

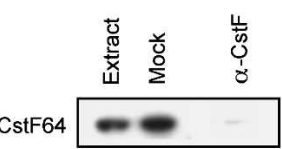

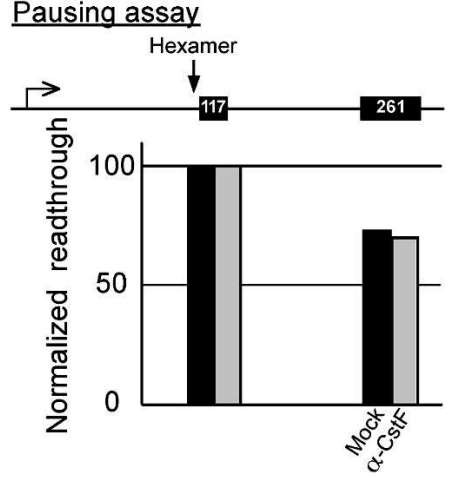

FIGURE 5. CstF is not required for poly $(A)$-dependent pausing/slowing in vitro. ( $A$ ) CstF is not required for poly $(A)$ signal-mediated pausing in vitro. The Western blot documents physical depletion of CstF and shows equal amounts of untreated extract, mock (anti-E1B)-depleted extract, and anti-CstF64 depleted extract run on an 8\% SDS-polyacrylamide gel and transferred to PVDF membrane (Roche) followed by overnight incubation with $\alpha$-CstF64 antibody 6A9. To visualize the blot, we used the BM Chemiluminescence Western Blotting Kit Mouse/Rabbit (Roche), and the blot was exposed to Hyper film (Amersham). We note that two CstF bands appear in this blot, previously attributed to different posttranslationally modified forms (Takagaki et al. 1990). However, two bands are not always apparent as seen in parts $B$ and $C$ and as reported by Wallace et al. (1999). The cleavage assay documents functional depletion of CstF. The RNA substrate was incubated with either mock or CstF depleted extract for $2 \mathrm{~h}$ followed by RNA isolation. The in vitro pausing assay was carried out by using a plasmid reporter closely related (and identical in the region mapped) to the pAP $\left\langle\mathrm{C}_{9}\right\rangle$ construct in Figure 5 of Tran et al. (2001). (B) Similar to $A$ except mock depletion was with normal goat antibody and probing was with 3A7. (C) Similar to $A$ but using the reporter of Figure $4 \mathrm{~A}$ and a G/U-less insert (insert iii, and also $v$ for normalization). Probing was with 3 A7.

of the assay. Although this assay cannot normally distinguish between poly(A)-dependent pausing and poly(A)dependent termination, in the special case of G/U-less
poly(A) signals that do not drive termination (Fig. 1, also Connelly and Manley 1988), it becomes a kinetic assay that specifically reports poly(A)-dependent pausing or slowing 
of the polymerase. Recall that with the nuclear run-on assay polymerase slowing in the steady state increases polymerase density over downstream cassettes, thereby increasing the downstream cassette signal. In contrast, polymerase slowing is predicted to decrease the downstream cassette signal in the in vitro kinetic assay. Thus, the Tran et al. (2001) assay is based on entirely different experimental assumptions from the run-on assay and therefore offers a genuinely independent assessment of the conclusion that the poly(A) signal hexamer directs the polymerase to pause.

Figure 4A shows the results of an in vitro assay that confirms the ability of the AAUAAA hexamer to slow down/pause the polymerase. Either an intact or mutant hexamer (Fig. 1B, inserts iv and v) was inserted into a reporter plasmid upstream of two G-less cassettes. The plasmid DNAs were then allowed to transcribe in HeLa nuclear extract for $15 \mathrm{~min}$, and the transcripts were digested with RNase T1 and run on a gel. The intensities of the resulting gel bands correspond to the number of polymerases that have transcribed to the end of the respective G-less cassettes. Lane 1 shows that after crossing a hexamer some, but not all, polymerases that transcribe the pre-cassette reach the end of the post-cassette during the time allowed for transcription. Lane 2 shows that a larger proportion of the polymerases reach the postcassette when they cross a hexamer that has been inactivated by mutation. Quantitation of these results shows that the proportion of polymerases making it from the precassette to the postcassette (the post/pre ratio) after crossing a wildtype hexamer is only $63 \%$ of the proportion that make it after crossing the mutant hexamer (Fig. $4 \mathrm{~B}$, gray bars). The simplest interpretation of these results is that the polymerases pause or slow down after crossing an AAUAAA hexamer, thereby reducing the number of polymerases that are able to reach the downstream cassette during the time allowed for transcription.

Interestingly, Figure $4 \mathrm{~B}$ shows that this effect is not greater for a full poly(A) signal than for the G/U-less poly(A) signals. This suggests that even for a full poly(A) signal it is pausing that dominates the output of the Tran et al. (2001) elongation assay (i.e., any termination in this assay occurs only subsequent to pausing). To confirm explicitly that the polymerases that fail to reach the second cassette are template-engaged polymerases that are moving more slowly, we analyzed ternary complexes isolated by size-exclusion chromatography. We then asked whether, after crossing an AAUAAA hexamer, there was an overrepresentation of nascent transcripts bearing precassettes but no postcassettes, as predicted for more slowly elongating polymerases. Note that because only ternary complexes are analyzed any polymerases that fail to reach the postcassette because of transcript release will not contribute to the analysis.

Figure 4, C and D, validates the size-exclusion method. Figure $4 \mathrm{C}$ confirms that elution of RNA in the excluded volume of a size-exclusion column depends on the integrity of the DNA. Two plasmids expressing different length Gless cassettes were separately transcribed to produce transcription complexes. One of these was then digested with DNase I, and then the two were mixed and separated by size-exclusion chromatography, and their transcripts separately detected by virtue of their G-less cassette sizes. Figure $4 \mathrm{C}$ reveals a peak of transcript in the excluded volume (fraction 8) only for complexes not digested by DNase I. Agarose gel electrophoresis (data not shown) confirmed that most or all of the intact DNA eluted in this same fraction. Figure 4D shows that RNA elutes with the DNA only if the RNA is a genuine participant in a ternary complex. When the RNA is cut free of the ternary complexes using RNase $\mathrm{H}$, little of the cut RNA $(\sim 800 \mathrm{nt}$ long) remains in the excluded volume (i.e., in fractions containing plasmid DNA). Moreover, the DNA in this experiment eluted mostly in fraction 6, with a small amount in fraction 7, and that is also the elution pattern of the small amount of transcript that escapes cutting by the RNase H. Therefore, to characterize the RNA in ternary complexes, we fractionated transcription mixtures by size exclusion and analyzed the RNA in the DNA-containing fractions.

Figure $4 \mathrm{E}$ shows the results of an in vitro pausing assay in which the analysis was carried out directly on ternary complexes isolated by size-exclusion chromatography. Agarose gel electrophoresis identified the DNA-containing fractions as numbers 7 and 8 (Fig. 4E, left). Cassette analysis of these fractions (as for Fig. 4A,B) confirmed that ternary complexes were more likely to bear transcripts containing an upstream cassette but lacking a downstream cassette if they had crossed AAUAAA hexamers. This confirms that ternary complexes travel more slowly after crossing a hexamer and are thus less likely to reach the cassette downstream during the time of the assay.

Note that the pausing/slowing summarized in the experiments of Figure 4, B and E, were measured, not at the position of the hexamer, but across a region or "window" (delineated by the distal ends of the two cassettes) that does not even begin until 120 or more bp downstream of the hexamer. Thus, in agreement with the run-on experiments, ternary complexes see the hexamer not as a localized pause site but as a signal that directs a change in their elongation properties over downstream DNA.

\section{CstF is not required for pausing in vitro}

Having established that the G/U-rich region of the poly(A) signal is not required for pausing, we turned our attention to CstF, which binds to the G/U-rich region. Although this RNA region is dispensable for pausing, it remained possible that CstF is nevertheless involved in the pausing, not through binding to the G/U-rich region itself but through its interaction with CPSF. As mentioned previously, CstF 
interacts with CPSF in vitro, and these two factors bind to the poly $(\mathrm{A})$ signal cooperatively (see Fig. 1A). Moreover, in vitro, after $3^{\prime}$-end cleavage is complete, mammalian CstF remains associated with the processed RNA in pull-down experiments (Rigo et al. 2005), suggesting that CstF and CPSF may indeed function as a pair even in the absence of a G/U-rich region.

To determine whether CstF is required for pausing, we attempted to use RNAi-mediated knockdowns of CstF. However, our knockdowns were insufficient to significantly affect function as determined by the efficiency of poly $(\mathrm{A})$ site processing in vivo. Therefore, we turned to immunodepletion of CstF from nuclear extracts followed by transcription elongation assays like those shown in Figure 4. The efficiencies of depletion obtained in these experiments is shown by the Western blots and cleavage assays in Figure 5, A and B. These depleted extracts were then subjected to in vitro pausing analysis as for Figure 4, $\mathrm{A}$ and $\mathrm{B}$. Figure $5 \mathrm{~A}$ shows that even after the functional removal of almost two-thirds of the CstF (cleavage assay), pausing/slowing remained equivalent to that for an intact poly(A) signal (histogram). Figure 5B shows that even after complete depletion of CstF (according to both Western and cleavage analysis), the CstF-depleted extract was still able to mediate substantial pausing/slowing by the polymerase (histogram). The slight decrease in pausing efficiency of the CstF depleted extract in Figure 5B may indicate coimmunoprecipitation of one or more important factors along with CstF.

We considered the possibility that the G/U-rich region and CstF may serve redundant functions so that the presence of either is sufficient to induce pausing. We therefore carried out a pausing assay lacking both CstF in the extract and a G/U-rich region in the poly(A) signal. The results are summarized in Figure 5C, and show that neither the $\mathrm{G} / \mathrm{U}$-rich region nor $\mathrm{CstF}$ is required for pausing/slowing of the polymerase. Thus, from all of these results, we conclude that pretermination poly(A)-dependent pausing can be uncoupled from the rest of poly $(A)$ signal function (i.e., processing and termination) by removal of any member of the G/U-CstF-CTD triumvirate (see Fig. 1A).

\section{DISCUSSION}

Poly(A)-dependent pausing occurs at a critical juncture in the production of a mature mRNA. The sequence of the message has been fully transcribed, but critical decisions about the fate of the RNA have not yet been made, and neither $3^{\prime}$-end processing nor termination has yet occurred. Indeed, poly $(\mathrm{A})$-dependent pausing occurs in the complete absence of either $3^{\prime}$-end processing (Fig. 2B) or transcription termination (Fig. 3B,C; Park et al. 2004). Perhaps pausing or slowing of the polymerase reflects the release of positive elongation factors, as suggested by chromatin immunoprecipitation experiments in yeast (Kim et al. 2004a). This may create a permissive environment for events that follow, or it may reflect checkpoint activity preceding commitment to processing and termination (Orozco et al. 2002).

In this report we have begun to dissect the biochemical basis of the poly(A)-dependent pause. First, we showed that pausing is the specialized output of just a portion of the canonical poly(A) signal - the AAUAAA hexamer. Both in vivo Figs. $(2,3)$ and in vitro Figs. $(4,5)$, one or two such hexamers in the absence of any natural sequences were sufficient to direct the polymerase to pause. Moreover, not only was the G/U-rich region of the poly(A) signal not necessary for pausing neither was the CstF that binds to it (Fig. 5). Thus, the poly(A)-dependent pause is basically an AAUAAA hexamer-dependent pause.

The effect of the AAUAAA hexamer on transcription is long lasting. In vivo the effects of the hexamer can persist unabated for more than $1 \mathrm{~kb}$ (Fig. 3B,C). In vitro the assay is designed so that only effects that persist for well over $100 \mathrm{bp}$ are detected (see below). This is most easily understood as a stable change in the elongation properties of the polymerase, consistent with a hexamer-induced release of positive elongation factors (Kim et al. 2004a) or the recruitment of a negative elongation factor. We have attempted to ascertain the role of CPSF in this process by using knockdown via RNAi in vivo or immunodepletion in vitro, but in our hands neither approach can effect a sufficient reduction in the level of CPSF to significantly affect even its canonical functions.

What is the relationship of AAUAAA-induced pausing to other pause elements that have been reported? At the in vitro level, we are not aware of any elements with the properties reported here for the AAUAAA hexamer. All elements of which we are aware behave in vitro as "pause sites," that is, their effect on the polymerase is completely local. Polymerases pause at the position of the element, but when they escape they resume transcription as before (e.g., Palangat et al. 2004). In contrast, the AAUAAA hexamer affects polymerase behavior for hundreds of base pairs down the template. Indeed, our in vitro experiments were deliberately designed to report on events only downstream of the hexamer. The rate of progress on the template from one cassette to another is measured by using pairs of cassettes that reside entirely downstream of the hexamer Figs. $(4,5)$ so that the slowing detected does not include any slowing that may also occur at the hexamer position itself. Thus, in vitro the hexamer induces a persistent change in the transcriptional properties of the polymerase.

In vivo the effects appear to be the same. When termination is abrogated by removal of either the G/Urich region or the CTD, polymerase occupancy on the DNA template rises after the poly $(\mathrm{A})$ signal and remains elevated for over $1 \mathrm{~kb}$ downstream. As discussed earlier, this obviously reflects a long-lasting change in the elongation properties of 
the polymerase. We are aware of only one other element with similar properties, a 158-bp sequence that gave rise to a 1.7-fold increase in polymerase density that persisted for $3.5 \mathrm{~kb}$ downstream (Fig. 6D in Peterson et al. 2002). However, in contrast to the AAUAAA hexamer, this effect could not be localized to any specific nucleotide sequence within the 158-bp element (Peterson et al. 2002).

There are many AAUAAA hexamers sprinkled throughout genomic DNA. What might be the response of the polymerase to one of these isolated hexamers? Perhaps such polymerases pause, move slowly down the template for some distance, and then, in the absence of transcription termination, recover speed owing to specific or nonspecific effects. Perhaps this is the effect on transcription of weak upstream poly(A) signals for genes that exhibit alternative polyadenylation - the polymerase decelerates, but if cleavage complex formation is not sufficiently vigorous to make it through the checkpoint (Rigo et al. 2005), the polymerase resumes speed.

\section{MATERIALS AND METHODS}

\section{G-less run on assay}

The G-less run on assay was carried out as in Orozco et al. (2002) with the modification that $0.5 \mu \mathrm{g} / \mu \mathrm{L}$ of heparin was included in the transcription reaction mixture to improve the signal to noise. Assays with the $\alpha$-amanitin-resistant CTD-less polymerase were as described by Park et al. (2004).

\section{In vitro transcription elongation assay}

This assay was carried out essentially as described by Tran et al. (2001) but in a total volume of $12.5 \mu \mathrm{L}$ of transcription mixture containing $0.3 \mu \mathrm{g}$ of plasmid DNA, $4 \mu \mathrm{L}$ of HeLa nuclear extract, and $10 \mu \mathrm{Ci}$ of $\left[\alpha-{ }^{32} \mathrm{P}\right] \mathrm{CTP}$. Typical final concentrations were $6.4 \%$ glycerol; $6.4 \mathrm{mM}$ Hepes (pH 7.9); $32 \mathrm{mM} \mathrm{KCl;} 64 \mu \mathrm{M}$ EDTA; $2.2 \mathrm{mM}$ DTT; $0.032 \mathrm{mM}$ PMSF; 5-6 mM MgCl $2 ; 6-8 \mathrm{mM}$ sodium citrate ( $\mathrm{pH}$ 6.7); $10 \mathrm{U}$ Anti-RNase (Ambion); $20 \mathrm{mM}$ creatine phosphate; $200 \mu \mathrm{M}$ ATP, UTP, and GTP; and $5 \mu \mathrm{M}$ cold CTP. $\mathrm{MgCl}_{2}$ and sodium citrate concentrations were optimized for each extract. Transcription was stopped by using $34 \mathrm{mM}$ EDTA followed by RNase T1 digestion (400U, Ambion) for $30 \mathrm{~min}$ at $45^{\circ} \mathrm{C}$. The RNA was isolated by using TRIzol/chloroform (GIBCO BRL), displayed on an $8 \%$ polyacrylamide gel, scanned on a BioRad Molecular Imager FX, and analyzed by using ImageQuant software (Molecular Dynamics).

\section{Size-exclusion chromatography of transcription reactions}

Fourfold transcription reactions were stopped by the addition of EDTA to $10 \mathrm{mM}, \mathrm{NaCl}$ to $1 \mathrm{M}$, and Sarkosyl to $0.5 \%$. The sample was then applied to a $3.1 \mathrm{~mL}(0.62 \times 211 \mathrm{~cm})$ Agarose A15M (BioRad) column was as described by $\mathrm{Gu}$ and Marzluff (1996) with some modifications and eluted with a buffer containing $6.4 \mathrm{mM}$ Hepes, $32 \mathrm{mM} \mathrm{KCl}, 1 \mathrm{M} \mathrm{NaCl}, 1 \mathrm{mM}$ EDTA, and $0.5 \%$ Sarkosyl. Total RNA and DNA were isolated from each fraction by phenol-chloroform extraction, recovered by ethanol precipitation, and then digested with RNase T1 for G-less cassette analysis or with Proteinase $\mathrm{K}$ in $0.5 \%$ SDS for analysis of intact RNA or DNA.

\section{In vitro poly(A) site cleavage of premade RNA}

Radiolabeled and capped (cap analog from Ambion) RNA was made by using T3 RNA polymerase (Stratagene). RNA containing the synthetic poly(A) signal (Levitt et al. 1989) was gel purified and 50,000 CPM of RNA was used for the cleavage assay employing standard procedures (Rigo et al. 2005).

\section{CstF depletion}

Thirty microliters of Protein G magnetic beads (Dynal) were washed with $150 \mu \mathrm{L}$ of $0.1 \mathrm{M}$ sodium phosphate buffer $(\mathrm{pH} 7)$. Following resuspension in $30 \mu \mathrm{L}$ of the same buffer, $36 \mu \mathrm{L}$ of culture supernatant for each of two $\alpha$-CstF 64 monoclonal antibodies (3A7 and 6A9) (Wallace et al. 1999) was added followed by incubation for $2 \mathrm{~h}$ at $4^{\circ} \mathrm{C}$. The beads were then washed with PBS followed by addition of $30 \mu \mathrm{L}$ HeLa nuclear extract and incubation for $2 \mathrm{~h}$ at $4^{\circ} \mathrm{C}$. Next the beads were magnetically selected, and the supernatant was transferred to a new antibody-bead preparation and incubated for another hour. Finally, the beads were magnetically removed, and the supernatant was frozen in liquid nitrogen. For Figure 5, B and C, the supernatant was scavenged of any free antibody by means of an additional 20-min incubation with $15 \mu \mathrm{L}$ of Protein G beads. For Figure 5A the antibodies were crosslinked to the Protein G beads according to the Dynal protocol, and the scavenging step was not used. For mock depletion we used either anti-E1B (2A6) (Dass et al. 2001) or normal goat antibody (Santa Cruz Biotechnology).

\section{ACKNOWLEDGMENTS}

We thank Frank Rigo for discussions during the course of this work; Clint MacDonald for the 3A7, 6A9, and 2A6 monoclonal antibodies; David Bentley for the $\triangle \mathrm{CTD}$ expression plasmid; Steve Kim for contributing to the initial stages of this work; and the NIH for grant GM50863.

Received April 4, 2006; accepted May 4, 2006.

\section{REFERENCES}

Ansari, A. and Hampsey, M. 2005. A role for the CPF $3^{\prime}$-end processing machinery in RNAP II-dependent gene looping. Genes \& Dev. 19: 2969-2978.

Bird, G., Zorio, D.A., and Bentley, D.L. 2004. RNA polymerase II carboxy-terminal domain phosphorylation is required for cotranscriptional pre-mRNA splicing and 3 '-end formation. Mol. Cell. Biol. 24: 8963-8969.

Bird, G., Fong, N., Gatlin, J.C., Farabaugh, S., and Bentley, D.L. 2005. Ribozyme cleavage reveals connections between mRNA release from the site of transcription and Pre-mRNA processing. Mol. Cell 20: $747-758$.

Calvo, O. and Manley, J.L. 2003. Strange bedfellows: Polyadenylation factors at the promoter. Genes \& Dev. 17: 1321-1327.

Chen, F., MacDonald, C.C., and Wilusz, J. 1995. Cleavage site determinants in the mammalian polyadenylation signal. Nucleic Acids Res. 23: 2614-2620. 
Connelly, S. and Manley, J.L. 1988. A functional mRNA polyadenylation signal is required for transcription termination by RNA polymerase II. Genes \& Dev. 2: 440-452.

1989. RNA polymerase II transcription termination is mediated specifically by protein binding to a CCAAT box sequence. Mol. Cell. Biol. 9: 5254-5259.

Dantonel, J.C., Murthy, K.G., Manley, J.L., and Tora, L. 1997. Transcription factor TFIID recruits factor CPSF for formation of 3' end of mRNA. Nature 389: 399-402.

Dass, B., Attaya, E.N., Wallace, A.M., and MacDonald, C.C. 2001. Overexpression of the CstF-64 and CPSF-160 polyadenylation protein messenger RNAs in mouse male germ cells. Biol. Reprod. 64: $1722-1729$.

Dichtl, B., Aasland, R., and Keller, W. 2004. Functions for S. cerevisiae Swd2p in $3^{\prime}$ end formation of specific mRNAs and snoRNAs and global histone 3 lysine 4 methylation. RNA 10: 965-977.

Dye, M.J. and Proudfoot, N.J. 2001. Multiple transcript cleavage precedes polymerase release in termination by RNA polymerase ii. Cell 105: 669-681.

Flaherty, S.M., Fortes, P., Izaurralde, E., Mattaj, I.W., and Gilmartin, G.M. 1997. Participation of the nuclear cap binding complex in pre-mRNA 3' processing. Proc. Natl. Acad. Sci. 94: 11893-11898.

Fong, N. and Bentley, D.L. 2001. Capping, splicing, and 3' processing are independently stimulated by RNA polymerase II: Different functions for different segments of the CTD. Genes \& Dev. 15: 1783-1795.

Fong, N., Bird, G., Vigneron, M., and Bentley, D.L. 2003. A 10 residue motif at the C-terminus of the RNA pol II CTD is required for transcription, splicing and 3' end processing. EMBO J. 22: 4274-4282.

Gavin, A.C., Bosche, M., Krause, R., Grandi, P., Marzioch, M., Bauer, A., Schultz, J., Rick, J.M., Michon, A.M., Cruciat, C.M., et al. 2002. Functional organization of the yeast proteome by systematic analysis of protein complexes. Nature 415: 141-147.

Gilmartin, G.M. and Nevins, J.R. 1989. An ordered pathway of assembly of components required for polyadenylation site recognition and processing. Genes \& Dev. 3: 2180-2190.

$\mathrm{Gu}, \mathrm{X}$. and Marzluff, W.F. 1996. 3' Processing and termination of mouse histone transcripts synthesized in vitro by RNA polymerase II. Nucleic Acids Res. 24: 3797-3805.

Hammell, C.M., Gross, S., Zenklusen, D., Heath, C.V., Stutz, F., Moore, C., and Cole, C.N. 2002. Coupling of termination, 3' processing, and mRNA export. Mol. Cell. Biol. 22: 6441-6457.

Kim, M., Ahn, S.H., Krogan, N.J., Greenblatt, J.F., and Buratowski, S. 2004a. Transitions in RNA polymerase II elongation complexes at the $3^{\prime}$ ends of genes. EMBO J. 23: 354-364.

Kim, M., Krogan, N.J., Vasiljeva, L., Rando, O.J., Nedea, E., Greenblatt, J.F., and Buratowski, S. 2004b. The yeast Rat1 exonuclease promotes transcription termination by RNA polymerase II. Nature 432: 517-522.

Levitt, N., Briggs, D., Gil, A., and Proudfoot, N.J. 1989. Definition of an efficient synthetic poly(A) site. Genes \& Dev. 3: 1019-1025.

Maniatis, T. and Reed, R. 2002. An extensive network of coupling among gene expression machines. Nature 416: 499-506.

Murthy, K.G. and Manley, J.L. 1995. The $160-\mathrm{kD}$ subunit of human cleavage-polyadenylation specificity factor coordinates pre-mRNA 3'-end formation. Genes \& Dev. 9: 2672-2683.

Natalizio, B.J., Muniz, L.C., Arhin, G.K., Wilusz, J., and Lutz, C.S. 2002. Upstream elements present in the $3^{\prime}$-untranslated region of collagen genes influence the processing efficiency of overlapping polyadenylation signals. J. Biol. Chem. 277: 42733-42740.

Orozco, I.J., Kim, S.J., and Martinson, H.G. 2002. The poly(A) signal, without the assistance of any downstream element, directs RNA polymerase II to pause in vivo and then to release stochastically from the template. J. Biol. Chem. 277: 42899-42911.

O'Sullivan, J.M., Tan-Wong, S.M., Morillon, A., Lee, B., Coles, J., Mellor, J., and Proudfoot, N.J. 2004. Gene loops juxtapose promoters and terminators in yeast. Nat. Genet. 36: 1014-1018.
Palangat, M., Hittinger, C.T., and Landick, R. 2004. Downstream DNA selectively affects a paused conformation of human RNA polymerase II. J. Mol. Biol. 341: 429-442.

Park, N.J., Tsao, D.C., and Martinson, H.G. 2004. The two steps of poly(A)-dependent termination, pausing and release, can be uncoupled by truncation of the RNA polymerase II CTD. Mol. Cell. Biol. 24: 4092-4103.

Peterson, M.L., Bertolino, S., and Davis, F. 2002. An RNA polymerase pause site is associated with the immunoglobulin $\mu$ s poly(A) site. Mol. Cell. Biol. 22: 5606-5615.

Proudfoot, N. 2004. New perspectives on connecting messenger RNA $3^{\prime}$ end formation to transcription. Curr. Opin. Cell Biol. 16: 272278.

Proudfoot, N.J., Furger, A., and Dye, M.J. 2002. Integrating mRNA processing with transcription. Cell 108: $501-512$.

Rigo, F., Kazerouninia, A., Nag, A., and Martinson, H.G. 2005. The RNA tether from the poly(A) signal to the polymerase mediates coupling of transcription to cleavage and polyadenylation. Mol. Cell 20: 733-745.

Ryan, K., Murthy, K.G., Kaneko, S., and Manley, J.L. 2002. Requirements of the RNA polymerase II C-terminal domain for reconstituting pre-mRNA 3' cleavage. Mol. Cell. Biol. 22: 1684-1692.

Sadowski, M., Dichtl, B., Hubner, W., and Keller, W. 2003. Independent functions of yeast Pcfllp in pre-mRNA $3^{\prime}$ end processing and in transcription termination. EMBO J. 22: 21672177.

Steinmetz, E.J. and Brow, D.A. 2003. Ssu72 protein mediates both poly(A)-coupled and poly(A)-independent termination of RNA polymerase II transcription. Mol. Cell. Biol. 23: 6339-6349.

Takagaki, Y. and Manley, J.L. 2000. Complex protein interactions within the human polyadenylation machinery identify a novel component. Mol. Cell. Biol. 20: 1515-1525.

Takagaki, Y., Manley, J.L., MacDonald, C.C., Wilusz, J., and Shenk, T. 1990. A multisubunit factor, CstF, is required for polyadenylation of mammalian pre-mRNAs. Genes \& Dev. 4: 2112-2120.

Tran, D.P., Kim, S.J., Park, N.J., Jew, T.M., and Martinson, H.G. 2001. Mechanism of poly(A) signal transduction to RNA polymerase II in vitro. Mol. Cell. Biol. 21: 7495-7508.

Wallace, A.M., Dass, B., Ravnik, S.E., Tonk, V., Jenkins, N.A., Gilbert, D.J., Copeland, N.G., and MacDonald, C.C. 1999. Two distinct forms of the $64,000 \mathrm{Mr}$ protein of the cleavage stimulation factor are expressed in mouse male germ cells. Proc. Natl. Acad. Sci. 96: 6763-6768.

West, S., Zaret, K., and Proudfoot, N. 2006. Transcriptional termination sequences in the mouse serum albumin gene. RNA 12: 655665.

Yeung, G., Choi, L.M., Chao, L.C., Park, N.J., Liu, D., Jamil, A., and Martinson, H.G. 1998. Poly(A)-driven and poly(A)-assisted termination: two different modes of poly(A)-dependent transcription termination. Mol. Cell. Biol. 18: 276-289.

Zhang, Z. and Gilmour, D.S. 2006. Pcfl1 is a termination factor in Drosophila that dismantles the elongation complex by bridging the CTD of RNA polymerase II to the nascent transcript. Mol. Cell 21: 65-74.

Zhang, Z., Fu, J., and Gilmour, D.S. 2005. CTD-dependent dismantling of the RNA polymerase II elongation complex by the premRNA $3{ }^{\prime}$-end processing factor, Pcf11. Genes \& Dev. 19: 15721580

Zhao, J., Hyman, L., and Moore, C. 1999. Formation of mRNA 3' ends in eukaryotes: mechanism, regulation, and interrelationships with other steps in mRNA synthesis. Microbiol. Mol. Biol. Rev. 63: 405-445.

Zhelkovsky, A., Tacahashi, Y., Nasser, T., He, X., Sterzer, U., Jensen, T.H., Domdey, H., and Moore, C. 2006. The role of the Brr5/Ysh1 C-terminal domain and its homolog Sycl in mRNA 3'-end processing in Saccharomyces cerevisiae. RNA 12: 435-445. 

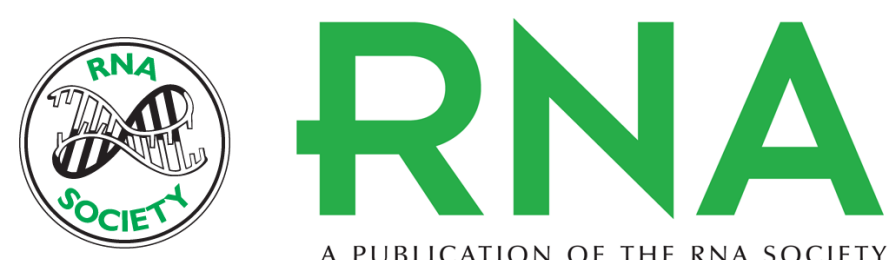

A PUBLICATION OF THE RNA SOCIETY

\section{The conserved AAUAAA hexamer of the poly(A) signal can act alone to trigger a stable decrease in RNA polymerase II transcription velocity}

Anita Nag, Kazim Narsinh, Amir Kazerouninia, et al.

RNA 2006 12: 1534-1544

References This article cites 45 articles, 29 of which can be accessed free at:

http://rnajournal.cshlp.org/content/12/8/1534.full.html\#ref-list-1

License

Email Alerting Receive free email alerts when new articles cite this article - sign up in the box at the Service top right corner of the article or click here.

To subscribe to RNA go to:

http://rnajournal.cshlp.org/subscriptions 